\title{
Exploring specific features of Transport Interchange Hubs (TIH) design, taking into account the climatic conditions of the Russian Arctic
}

\author{
Ilya V. Dunichkin'a ‘[0000-0001-9372-0741]', Clarice Bleil de Souza 2a ‘[0000-0001-7823-1202]' \\ Konstantin Bogachev ${ }^{1 b}$ '[0000-0003-0040-9765]', Camilla Pezzica ${ }^{2 b}$ '[0000-0002-0512-7591]'

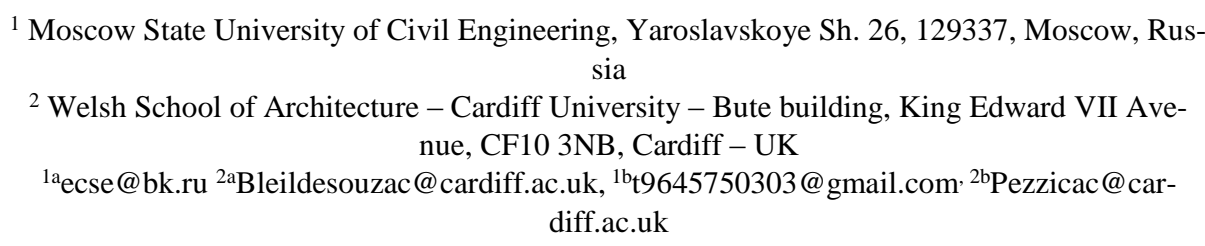

\begin{abstract}
This paper provides a more detailed analysis of the context of designing Transport Interchange Hubs (TIHs) in the Arctic Zone of the Russian Federation (AZRF). It uses a design framework proposed by another paper also submitted to his conference by the same authors [1] to discuss how green spaces can be integrated to $\mathrm{TIHs}$ in extreme climates to enhance the qualities of different types of spaces inside terminals considering implications of these in the overall assessment of building performance. It also discusses, the way pedestrian flow and movement is assessed and used in the design of TIH in the AZRF through advanced analysis techniques and how these could potentially be integrated with parametric design tools, finishing by considering the complexities involved in designing compact buildings, a necessary requirement to reduce heat losses and the impact of building footprint on the permafrost.
\end{abstract}

Keywords: Transport Interchange Hubs (TIH), Arctic, Arctic zone of the Russian Federation, Sustainable urban development, pedestrian flow, indoor landscaping, simulations.

\section{Introduction}

The Russian Arctic hosts cities since the $16^{\text {th }}$ century and have been sustained by intensive investments from the Soviet times to date, including the revival of the Northern Sea Route which is expected to foster and increase economic development in the region [2]. Within this vast territory and extreme climate, with temperatures reaching an average of $-30^{\circ} \mathrm{C}$ in winter, cities tend to be generally small in size and isolated from one another. Their survival and economic viability is dependent upon the exploitation of 
mineral resources and, due to their peculiar functioning mechanism, they host populations with a high turn-over, with an average migration index of 9.8\% [2]. However, despite these parallel and dynamic population expansion and contraction phenomena, cities in the Arctic Zone of the Russian Federation (AZRF) are in a state of (instable) equilibrium that makes them generally resilient to fluctuations in economic development, i.e. when development goes negative, people can easily leave whereas when conditions improve, people tend to come back. Long-term resilience is hence perhaps explained by seasonal migration. People in the Arctic undertake regular trips to Southern Russia due to fix-term business contracts; since the Soviet times they are granted governmental subsidies to travel to the South to maintain family and business ties, meaning they have a 'double-territorial identity' divided between the Arctic itself and the different parts of the country they also feel they belong to. These unique characteristics of migration in the AZRF mean that mobility needs to be preserved as a 'safety valve' to cope with economic dynamic fluctuations and to connect the North and South of the country [2]. Supporting mobility means investing in building and refurbishing transport networks, infrastructure and related facilities.

Transport is a serious issue in the Russian Arctic. It has implications in urban and social sustainable development as well as economic growth. Despite technological improvements in design and operation of passenger transport systems, total travelling time, in the AZRF remains significant; varying within 40 -100 minutes (for short distances), depending on the size of the settlement, weather conditions and the number of available modes of transport. This time is directly affected by the necessity to travel long distances within a vast territory as well as a lack of coordination among different modes of transport reaching interchange terminals. The combination of these two factors can result in, on average, $12 \mathrm{hs}$ waiting time within terminal buildings, meaning that any improvement targeted at reducing total travelling time by increasing the speed of vehicles along the journey, may be ultimately ineffective, due to the significant amount of time spent by passengers in TIHs during transfers. As a result, passengers' experience is strongly influenced not only by the time spent on the trip, but also by the quality and length of waiting time during their travel across extreme climate zones.

Compensating for waiting time, is a critical design requirement in TIHs around the world and has been in the agenda of several European projects in the recent years such as for instance: HERMES [3]; City HUB [4]; NODES [5]; Alliance [6]. Addressing this requirement normally involves providing several facilities and amenities to travellers such as offering convenience shopping, food and refreshments, 'workstations', etc. [7$11,12]$. These opportunities can be exploited by non-travellers and business managers through the design of more 'interesting' terminals, which can fulfil travellers' needs as well as act as community hubs, benefiting its customers (the travellers), and the city as a whole. In this context, TIH are seen not only as a host of pedestrian flow while people move from one mode of transport to another but also as a community centre for intercity passengers [13].

However, to date, implemented projects of terminals and stations in AZRF settlements have not taken into account the specifics of a market economy. They have not exploited fully opportunities which terminals could provide to introduce retail spaces, domestic hotels, 'interesting' waiting rooms, etc. while fulfilling the requirements and 
related design parameters for guaranteeing comfortable and functional human flows through universal design. Contrarily to the literature on TIHs in the European continent, Midland of Russia and Japan, which lately has seen more research in the area of customer satisfaction, TIH design strategies adopted in the AZRF have not yet properly integrated passengers' flow inside terminals with opportunity spaces to improve the quality of their waiting time. They also do not take properly into account the movement of people with limited mobility (PLM) inside terminals. To the best of the authors' knowledge there are no reports on customer satisfaction surveys among users of TIHs in the AZRF targeting terminals' design nor quantitative methods and models to predict and assess the integration of pedestrian flows within terminals with interior opportunity spaces.

This paper uses the methodology proposed by another paper submitted to this conference [1], to analyse in more detail passengers' movement within terminals as well as potential ways to explore design opportunities that would enhance the quality of passengers' waiting time. Movement is discussed in terms of how passengers' flow is currently simulated in the design of TIH in the AZRF [14] in contrast with how it could potentially be enhanced by borrowing some complementary analysis methods from Western Europe and Japan, whereas waiting time is exploited in terms of leisure opportunities potentially provided by TIHs once designed as community hubs suitable to operate in a harsh climate environment.

\section{Methodology}

In the paper [1] also submitted to this conference, the authors proposed a framework to address travellers' needs in the design of TIH using the Axiomatic Design (AD) method [15]. In this method, travellers' needs are translated into TIH functional requirements and subsequently into design parameters, to structure the development of a set of design specifications which can be used to more holistically take into account the integration of passenger flows and leisure opportunities provided to compensate for waiting times within TIH.

When applying this method, terminal buildings are understood as buildings, which provide shelter to mainly three different types of spaces:

(i) Decision spaces, comprising places within the terminal in which decisions take priority (e.g. buying a ticket, gathering information about transfer, passing a control point, etc.);

(ii) Movement spaces, in which passengers flow from one decision space to another, between different transport modes, or from a decision space to a transport mode;

(iii) Opportunity spaces, in which neither movement nor decision-making take priority and can be used to compensate for travellers' waiting time.

Under this framework, TIH become buildings, hosting passenger flows from different set of origin-destination pairs surrounded by opportunity spaces compensating for waiting time. The breaking of internal spaces into three different components is adaptable to different building scales, enabling the design of simple stations up to the design 
of complex buildings. This type of adaptability is important in designing TIHs in the AZRF as cities vary in size and terminals need to accommodate for fluctuations in population. Having TIHs as buildings which mainly host passenger flows also aid in focusing of safety and planning efficiency, two important design considerations in extreme climates which should take into account low temperatures and the risk of frostbite during emergency evacuation of passengers [16].

Section 3 focuses on discussing how green community areas can be designed as part of opportunity spaces in harsh climate zones, as in the case of AZRF. It is anticipated that this would improve the (costumer) experience associated to long waiting times by offering a positive opportunity for people to gather around and interact with nature. Sections 4 and 5 of this paper, will explore in more detail how movement spaces are currently embedded in the design of terminals in the AZRF and how simulation methods more commonly used in Western Europe could potentially add to the design of these spaces, from improving visual communication and information effectiveness up to the development of more compact layouts.

\section{Indoor gardening: an extra attraction to compensate for waiting time and organize human flow}

A characteristic feature of cities in the AZRF is their targeted provision of gathering spaces. These spaces were considered strategic in the development of Arctic cities in Russia as they contribute to maintain population's mental well-being under extreme climatic conditions of low temperature, snowdrifts, strong winds and stand long periods of darkness during the winter [17]. Community spaces in the AZRF are normally provided as theatres, libraries, clubs and sport facilities (mainly in large cities), indoor activities which can function throughout the long and extreme periods in which going outdoors is inhospitable or unsafe. The use of indoor landscaping as a core for community gathering is underexplored. However, once considered too expensive indoor green spaces are now more affordable through new technologies [18]. This opens an interesting opportunity for the design of TIH in which compensating for waiting time by enabling/promoting the distribution and use of green spaces can also aid in creating community hubs.

Green elements can be central in promoting well-being and community integration, even more so in extreme climates such as the one of the AZRF where they are quite rare. They can "overcompensate or generate artificial interiors that normalize conditions, reverting them to familiar temperature latitudes where most people live" [17] thus enabling a smoother transition from one condition to another during seasonal migrations. Their viability can be justified by the need to enhance travellers' experience, qualitatively improving several types of opportunity spaces and by aiding in temperature and moisture regulation of indoor environments that they would produce in return. Landscaping features can be adequately disposed alongside movement spaces and/or combined with amenities (such as cafes, restaurants, etc.) as well as waiting rooms and 
hotels, promoting sustainable architecture and developing TIH as multifunctional public spaces [19]. Besides that, they can be deployed as specific attractions in atriums acting as internal gardening and supporting social interactions.

Gardening inside and outside TIH can help to make the public space more sustainable, safe and educational [20]. However, considering landscaping as a key component of opportunity spaces alongside movement spaces implies assessing their influence in design decisions related to location and direction of pedestrian flows. Additionally, one of the first design decisions to make concerns if these spaces will be disconnected from the climate, i.e. operated and controlled artificially, or if they will be climate adaptive and therefore potentially have seasonal use only. This suggests that, functional requirements of these spaces should be carefully crafted in relation to how they can potentially contribute to improve the experience of travellers passing through movement spaces and/or be used as opportunity spaces themselves by fulfilling specific community needs and/or hosting specific types of social activities.

From these set of functional requirements, design parameters such as the following can then be defined, to support these spaces' distribution, shape and sizing:

- Linear landscaping for pedestrian malls, aboveground crossings and concourses;

- Group landscaping of recreation areas, halls, atriums, foyers, lobbies;

- Solitary-dot landscaping of recreations, halls, atriums, foyers, lobbies;

- Systematic landscaping of recreations, halls, atriums, indoor areas for baggage claim;

- Vertical landscaping in separately standing vertical gardening modules and pots on walls, pillars and columns.

Related maintenance considerations need to be factored in the design specification as design constraints to allow irrigation, sanitation, replacement, and pruning. These constraints cannot interfere with movement spaces as this could disrupt pedestrian flow by creating risks for travellers' safety, obstructing visibility and hindering internal wayfinding. BIM can provide an initial environment for safety analysis and the detection of functional clashes [21] and once connected to dynamic thermal simulations (to verify the potential for these spaces to provide thermal comfort), space syntax, and pedestrian flow simulations aid in improving the quality of design specifications. The design of green spaces and of the surrounding areas has hence to be perfectly integrated as to satisfy their programmatic use.

Different types of physical and cognitive interactions between green spaces and pedestrian flows can be assessed through pedestrian flow simulations and Space Syntax analysis as part of Environmental Psychology studies. Space syntax can help in creating enclosed green spaces that trigger small or larger groups' social interaction (e.g. by using the Clustering index), promote safety through co-awareness (Connectivity index), invite people to stay in a place where they can look at others' (Integration index); which means that it could help quantifying the 'Interaction' qualities of green pockets [22]. Also, Space Syntax can be used to explore space configurations in 3D, therefore accounting for the visual integration of vertical landscaping in the design and its influence on the visibility of vertical signalling [23]. 
To the best of the authors' knowledge, there is no simulation tool comprehensive enough to deal simultaneously with these different simulation domains, meaning the effective deployment and distribution of green spaces inside TIHs calls for more research related to integrated software development. Current parametric design environments (e.g. Dynamo for Revit or Grasshopper for Rhyno) tend to integrate different types of building performance assessment methods (airflow, thermal, daylight, spatial etc.) but are not sufficiently well connected to pedestrian flow simulations or Space Syntax.

\section{Pedestrian flow in TIHs to assess and inform design}

Quantitative methods and simulation tools in general are not sufficiently used to predict and simulate the dynamics and interactions of passengers' flows in TIHs in the AZRF and, in the Western Europe, they started being used quite recently [5]. If added to that, one considers there is insufficient unified automated control system of the transport complex and a lack of electronic data available for passengers about the network in general, improvements in relation to total travel time in AZRF become limited [24]. In most cases, the focus tends to be on design parameters related to transport network demands, mainly to rationalize infrastructure costs and reduce vehicle travel time. However, 'soft' design parameters such as those listed in table 1 should be used for specific tasks such as to configure key connections within terminal spaces as well as the links between terminals and the city.

Connections are central in the design of TIH buildings and can be initially analysed in terms of hybrid origin-destination / space representations as proposed in [1]. However, connections should be designed not only according to transport safety and efficiency but also with regards to enabling cognitive ease during spatial navigation, effortless movement and environmental comfort, which can be seen as a holistic requirement including adequate temperatures up to a pleasant journey [25]. Besides that, connections should be adequate to effectively accommodate elderly and people with disabilities, promoting social inclusion and well-being through movement without borders for all.

Table 1. Design parameters for movement spaces within TIH buildings.

\begin{tabular}{lll}
\hline $\begin{array}{l}\text { Type of } \\
\text { connection }\end{array}$ & $\begin{array}{l}\text { Design parameters for access / entry } \\
\text { points to terminal }\end{array}$ & $\begin{array}{l}\text { Design parameter for movement } \\
\text { spaces inside the terminal }\end{array}$ \\
\hline \multirow{2}{*}{ Pedestrian } & $\begin{array}{l}\text { Sidewalks, pedestrian areas and squares, } \\
\text { alleys, landscaping areas, entrance and } \\
\text { exit from the open parking lot, etc. }\end{array}$ & $\begin{array}{l}\text { Horizontal - paths, corridors, pe- } \\
\text { destrian malls } \\
\text { Vertical - Escalators, lifts, stairs, } \\
\text { ramps, }\end{array}$ \\
Vehicles & $\begin{array}{l}\text { Open Parking, drop-off area, bus stops, } \\
\text { private hired vehicles area, etc. }\end{array}$ & $\begin{array}{l}\text { Underground and surface parking, } \\
\text { entrance groups, ramps, car lifts, }\end{array}$
\end{tabular}


The final shape and size of the aforementioned design parameters should primarily account for the density, speed and direction of flows considering a wide range of special cases. A commonly employed method prescribed by statutory authorities and building regulations in Russia is the empirical formula, based on a gas/hydrodynamic analogy, proposed by Predtechesky and Milinskii [26] which relates speed, density of flow and path capacity with each other and therefore is able to predict how flow characteristics change in relation to different widths and route types, how crowding and bottlenecking happen in a path with insufficient capacity as well as how convergence and divergence, merging and branching of flows can be determined. Despite being easy to use and requiring very simple computation, the method does not take into account the non-homogeneity of flow speeds, which means for instance it cannot account for the needs of people with limited mobility (PLM), nor "special cases of pedestrian flow (e.g. movement at high density conditions, crossing flows and contra-flows, etc.)" [26]. A more advanced method is proposed by Kholshevnikov [26-27] who investigates the effect of emotional state and nature of pathway in travel speed stating that once a threshold density is exceeded, flow speed will change. Proposals for connecting travel speed with people's physical ability and density of crowd enabling a series of considerations on how these affect cross flows, contra-flows, movement in routes with unlimited width and movement through openings are outlined. More advanced and stochastic model are developed to relate travel speed with inter-person distance and density of flow.

Gas-hydrodynamic analogy models are good for assessing high density crowd behaviour, such as in emergency situations. They are also suitable to cope with situations in which pedestrians respond to group forces rather than individual needs. However, they are not really suitable to cope with pedestrian undertaking random decisions, e.g. adopting alternative routes within the building by going shopping instead of going to the platform, etc. Thus, for instance a massive outflow of visitors to the TIH shopping areas would load pedestrian mobility interchanges to the utmost and creates traffic jams already at the exit from the parking lot, significantly overloading those located around the street. The flow of people in such a situation would provoke a discomfort for those reaching the terminal because the sizing of pathways was based on regulations and standards for building evacuation which do not consider the fact that people can go to the TIH for shopping. Essentially, pathways will be geometrically sized and shaped based on the former rather than also taking the latter into consideration.

The gas-hydrodynamic analogy is a basic tool in aiding design assessment and analysis of pedestrian flow in large buildings [27] meaning the method can be used to suitably size both peak and average flows within TIHs. With insufficient substantiation of the design solution, design proposals do not withstand the extreme load of human flow and consequently density thresholds are rapidly exceeded and crowding or queuing conditions occur. This, instantly reduces the perceived comfort of the TIH space for travellers, increasing total travelling time, and furthermore, creates a threat to the safety of children and people with limited mobility. Therefore, permissible density should be determined for both peak and average flows, so the pathway with lowest carrying capacity among the following can be adequately sized considering:

- Flow from local transportation to the TIH;

- Flow from long-distance routes to the TIH; 
- Flow within the TIH (from decision space to transport, between transport modes and between decision spaces);

- $\quad$ Flow from targeted visits to TIH related functions (commercial premises).

However, the estimation of pedestrian flow density is a non-trivial issue because empirical flow density estimations show large variations and lack consistency [26]. An acceptable approximation for flow density estimation is obtained when using the G.F. Voronoi algorithm [28] due to their capacity of generating an optimised subdivision of spaces into sub-regions whose boundaries are organically defined depending on the distribution of seeds points. The algorithm can be applied at any scale, meaning Voronoi diagrams could also be used to optimize the configuration of existing TIH plans having irregular morphologies [29]. Overall, using a Voronoi diagram could be a reasonable strategy to address problems related to facility location and zoning of elements within buildings as well as in cities as this method works with geometric proximity to trace regions of influence in space. Using simulations to aid space distribution with shortest connections can be extremely effective especially when the aim is to minimize the total length of movement spaces inside the building. This can be achieved either through simulations (e.g. via using Voronoi algorithms as described above) or through physical models such as those used by for instance Frei Otto to find minimal surface, following a well-known form-finding optimisation method [30].

Connecting the previously mentioned Voronoi algorithm, Voronoi diagrams or even more complex form finding principles with the results of the gas-hydrodynamic analogy to calculate and then appropriately accommodate pedestrian flows in TIH buildings suggests a clear route to use parametric analysis and parametric/generative design tools to find original and more effective design solutions for TIHs.

Gas-hydrodynamic analogy models are considered macroscopic flow models as in these models "individuals have no autonomy neither to change their kinematic state nor to control their interactions" [34]. They are appropriate to size occupancy and capacity in relation to maximum flow and speed, meaning they can be easily applied to building design and assessment and also connected to parametric design tools as suggested in the aforementioned paragraph. On the other side of the spectrum, microscopic models "consider that each individual can control her own dynamics and can recreate with accuracy specific local interactions (collision, overtaking) or model individual interest or preferences" [31], yielding potentially different design solutions. For instance, original results were obtained in a study considering a system of "agents" moving according to an algorithm that models the behaviour of birds, which in nature move in group following approximately one unique direction. This "swarm urbanism" approach was tested in a parametric design experiment to revamp the city of Melbourne in 2009 (see Fig. 1a) [32]. Furthermore, for the parametric design of the Taipei street network, Annie Chan and Yikai Lin from the University of Southern California [33] have proposed the use of Ants colonies' movement in a modified "swarm logic" approach (see Fig. 1b). Both methods are transferable to the design of TIHs to investigate 'logical' and preferred paths informing the internal layout of movement spaces and commercial software based on microsimulations are already available and being used in TIH refurbishment across Western Europe [5, 11]. 


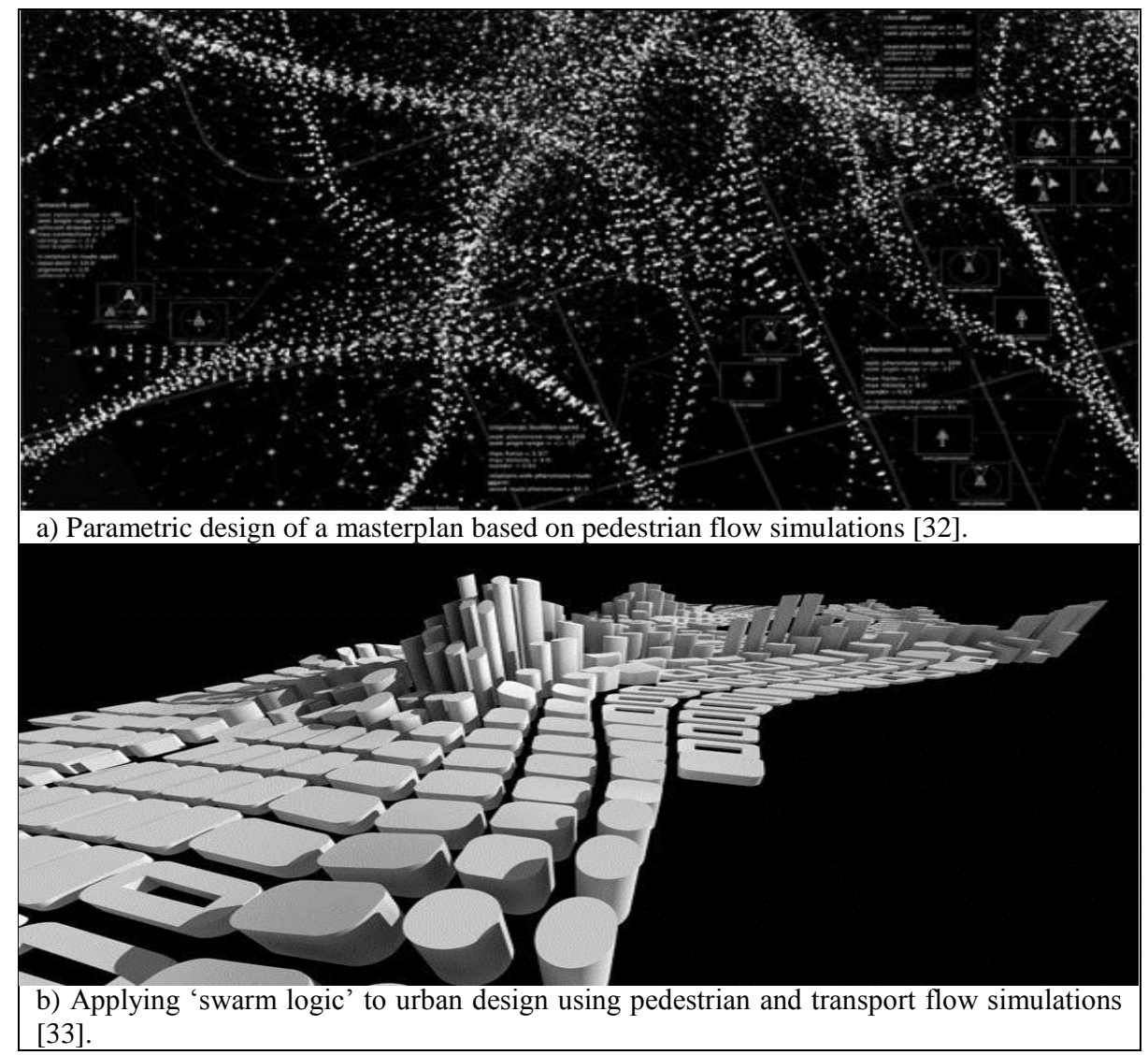

Fig. 1. Using agent based simulations in pedestrian movement and road network

Grounding TIH Planning decisions on the results of such simulation has the potential to deliver optimal performance and provide control to designers in relation to how design decisions affect pedestrian flow [34], empowering design experiments and aiding in the search for evidence-based design solutions. This is particularly important in the case of designing TIHs in the Arctic in which further constraints in relation to building compacity and safety need to be applied.

\section{The impact of compactness in pedestrian flow}

The analysis of pedestrian flow becomes particularly complex once compactness is stated as an essential functional requirement of TIHs in harsh climates to reduce heat losses and minimize impact on the permafrost through a contained and optimized building footprint [35]. Contrarily to western Europe and Japan in which waiting areas are controlled within the comfort zone $\left(18^{\circ} \mathrm{C}\right.$ to $\left.24^{\circ} \mathrm{C}\right)$, circulation spaces are controlled 
around up to $15^{\circ} \mathrm{C}$ and platforms are left uncontrolled, in the AZRF all spaces need to be controlled. Spaces of permanence such as sitting area should be kept within the comfort zone (between $18^{\circ} \mathrm{C}$ and $24^{\circ} \mathrm{C}$ ); circulation spaces need to be controlled between $0^{\circ} \mathrm{C}$ and $5^{\circ} \mathrm{C}$ and platform areas conditioned between $-15^{\circ} \mathrm{C}$ to $0^{\circ} \mathrm{C}$ to guarantee passengers safety. Once constraints related to building on the permafrost are added to that, such as compact and large building footprints with concentrated utility point built on pile foundations, considerations related to space distribution will forcefully need to be examined three dimensionally, i.e. by exploring multi-level space distributions.

Examples of multi-level space distributions include for instance the one presented in Figure 2, with parking area, roads and bus lines, trolleybuses, trams, recreational spaces with indoor landscaping in the lower tier and shopping and leisure areas in the middle tier, and train station, railways in the upper tier.

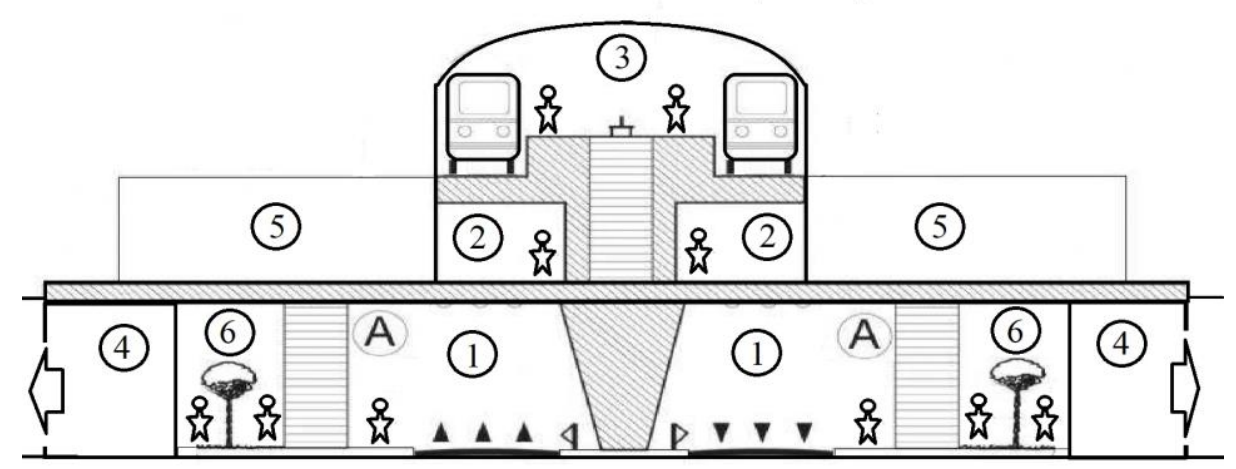

Fig. 2. Theoretical model of TIH: 1) Stopping points of urban ground-based passenger transport; 2) Vestibules station; 3) Railway station; 4) Car parking; 5) Distributive pedestrian level. 6) Green Vestibules for human flows.

The gas hydrodynamic analogy model is particularly useful in sizing pathways in this case because it does take into consideration different scenarios including not only different types of horizontal routes but also non-planar ones such as staircases, ramps, etc. under different circumstances of use (peak, average, emergency, etc.). TIHs with multi-level space distributions would therefore have pedestrian pathways sized similarly to entertainment complexes or stadiums, which require attention when assessing pedestrian flows in emergency situations and when restrictions are imposed on entrances for screening passengers and visitors due to different types of security requirements. In this type of space distribution, not only the sizing needs to happen in relation to full building evacuation but also the positioning of non-planar pathways and entrances. This means, for instance, entrances in the first level, stairs, escalators etc. need not only to be sized based on full building evacuation but also strategically positioned in relation to fastest exit routes, adding complexity to the layout of movement spaces 
calling for safety constraints and requirements related to different pairs of origin-destination to be considered together.

Particularly in multi-level space distributions, pedestrian flows become stable if enough space is given to entrances and if there is at least a duplication of vertical pathways between different levels (typically stairs plus elevators or similar automated systems). Despite more complex in terms of the sizing and layout of movement spaces, multilevel TIHs enable an efficient reallocation of pedestrian flows and better possibilities of exploring building centrality, i.e. developing key nodal spaces, potentially aiding the distribution of opportunity spaces provided with catering, trade, places for quiet rest, etc. (Fig. 2). Pedestrian and transport areas with parking lots are at different levels and do not intersect, resulting in a barrier-free and more protected environment all. In addition, a rational distribution of movement spaces towards key nodal spaces are easier to be conditioned at separate temperatures, enabling the latter to be more comfortable environments for social interaction. Multi-level systems are also an interesting option in refurbishment of existing facilities as they enable incremental upgrading through the creation of multilevel platforms staging construction, which in the Arctic can only happen during the brief warm season, and facilitating internal conditioning.

\section{Conclusions}

This paper aimed to explore specific requirements and related features of TIH design in the AZRF. Fitting within a design method developed in another paper [1] by the same authors, it explored in more detail the use of green spaces as an extra-attraction on compensating for waiting time as well as methods to simulate human flow within terminals to improve travelers' comfort and speed, thus aiding in reducing total travelling time. It also discussed the complexities involved in the adoption of a multi-level schematic layout which on the one hand increases building compactness but on the other hand affects flow density and increases the complexity of design assessment.

The study suggests the use of green spaces inside TIH not only improves comfort and well-being promoting the creation of pleasant community spaces, but can also positively contribute to directing pedestrian flow. When designed together with movement spaces, they can aid in the organization and distribution of pedestrians and, if carefully crafted, influence flow density and speed as well as support the organization of movement for people with limited mobility. Further studies are needed to better address cognitive interactions between green spaces and pedestrian flow. Space Syntax can aid to that and would need to be integrated to pedestrian flow simulations together with dynamic thermal modelling to properly assess holistic performance of green spaces and their contribution in overall building performance.

The study also shows that rationalizing the distribution of movement spaces based on origin-destination alone is far from sufficient to design safe, functional and effective $\mathrm{TIH}$ as these paths need to be carefully set up and sized with support of pedestrian flow simulations. Different models were discussed in section 4, from macroscopic models, based on the gas-hydrodynamic analogy, as recommended by Russian regulations and 
standards up to microscopic models such as agent based simulations which recently started being adopted to assess station refurbishment in Western Europe. Particular emphasis was given to the impact of compact layouts in pedestrian flow in which greater attention should be paid to the sizing of entrances and exits' points as well as sizing and positioning of vertical pathways. However, multi-level spatial layouts are highlighted as beneficial not only in terms of diminishing heat losses and minimizing impact on the permafrost but also for facilitating vehicles and vehicle / pedestrian segregation, and concentrating the distribution of opportunity spaces in key nodal points favoring indoor environmental control.

Further studies are necessary to better clarify which models are appropriate to be used in aiding design decisions and design assessment of different types of TIHs, considering not only building size and capacity but also its function in relation to the city. As pedestrian flows goes beyond simple internal origin-destination movement, a suitable choice for sizing and optimizing layout in TIHs designed to respond to neighborhood needs might be different than the ones used to size and design TIHs which act like city hubs. In addition, modelling of pedestrian flows still seems limited in accounting for the needs of people with limited mobility with regards to how they affect and are affected by flow density and speed. The same can be said in relation to accounting for movement of people in winter clothes, typical in AZRF, preventing functional requirements to be appropriately defined in relation to how these groups of people should be taken into account in design solutions (e.g. wider pathways, segregated routes, etc.).

Besides modelling choices, fine-tuning is still necessary to better integrate pedestrian flow models to the design process considering not only their use in parametric design but also their integration with other models such as for instance thermal simulations and space syntax so more integrated design solutions can be proposed beyond the context of the Russian Arctic.

\section{$7 \quad$ References}

1. Bleil de Souza, C., Dunichkin, I.V., Pezzica, C. A user-centred approach to design Transport Interchange Hus (TIH): A discussion illustrated by a case study in the Russian Arctic. International Conference on Computational Science and its Applications. Under review (2019).

2. Zamyatina, N., Goncharov, R., Population mobility and the contrasts between cities in the Russian Arctic and their southern Russian counterparts. Area Development and Policy 3(3) 293-308. (2018)

3. HERMES - High efficient and reliable arrangements for crossmodal transport, https://cordis.europa.eu/project/rcn/93149/reporting/en, last accessed 2019/02/10

4. City-HUB, http://www.cityhub.imet.gr/, last accessed 2019/02/10

5. NODES Interchanges - New tools for designing and operation of urban transport interchanges, http://www.nodes-interchanges.eu/, last accessed 2019/02/10

6. Alliance - Enhancing excellence and innovation capacity in sustainable transport interchanges, http://alliance-project.eu/, last accessed 2019/02/10

7. Hickman, R., Chen, C.L., Chow, A., Saxena, S.: Improving interchanges in China: the experiential phenomenon. Journal of Transport Geopgraphy, 42. pp. 175-186 (2015).

8. Hernandez, S., Monzon, A., Ona, R.: Urban transport interchanges: A methodology for evaluating perceived quality. Transportation Research Part A 84, pp. 31-43 (2016). 
9. Tsami, M., Adamos, G., Natrhanail, E., Budilovich, E., Yatskiv, I., Magginas, V.: A decision tree approach for achieving high customer satisfaction at urban interchanges. Transport and Telecommunication, 19(3), 194-202 (2018).

10. Monzon, A., Alonso, A., Lopez-Lambas, M.: Joint analysis of intermodal long distance-last mile trips using urban interchanges in EU cities. Transportation Research Procedia 27, pp. 1074-1079 (2017).

11. Booth, R.: D3.3.1 Identification and specification of the key areas of interchange design. In: Hoogendoom, C. (eds.) NODES 'New tools for Design and Operation of Urban Transport InterchangeS'. Project Report. European Commission, DG Research and Innovation (2015).

12. Danilina, N., \& Vlasov, D. Aspects of transport transit hubs construction management in coordination with object lifecycle projecting. In MATEC Web of Conferences (Vol. 86, p. 05017). EDP Sciences. (2016).

13. Vlasov, D., Danilina, N., \& Shagimuratova, A. The Priority Directions of Public Transport Transit Hubs Development on Commuter Railways. In Energy Management of Municipal Transportation Facilities and Transport (pp. 299-309). Springer, Cham. (2017).

14. Dunichkin, I. V.: Transport interchange hubs under the conditions of the Far North. In: Murgul, V. and Popovic, Z. (eds.) INTERNATIONAL SCIENTIFIC CONFERENCE ENERGY MANAGEMENT OF MUNICIPAL TRANSPORTATION FACILITIES AND TRANSPORT EMMFT, pp. 446-452. Advances in Intelligent Systems and Computing 692, Khabarovsk (2017).

15. Suh, N. P.: Axiomatic Design: Advances and Applications. Oxford University Press, New York (2001).

16. Kholshchevnikov, V., Korolchenko, D., \& Zosimova, O. Efficiency evaluation criteria of communication paths structure in a complex of buildings of maternity and child-care institutions. In MATEC Web of Conferences (Vol. 106, p. 01037). EDP Sciences. (2017).

17. Jull, M. Toward a Northern Architecture: The microrayon Arctic urban prototype. Journal of Archietctural Education 70(2) 214-222. (2016).

18. Korol, O., Shushunova, N., Lopatkin, D., Zanin, A., \& Shushunova, T. Application of Hightech Solutions in Ecodevelopment. In MATEC Web of Conferences (Vol. 251, p. 06002). EDP Sciences. (2018).

19. Esaulov, G.V. Sustainable architecture: from approaches to strategy of development. Vestnik of Tomsk State University of Architecture and Building. English version appendix, (2), 5-17. (2014).

20. Krasheninnikov, A. Structure of social space in pedestrian realm. Architecture and modern information technologies (4), 21. (2012).

21. Cherkina, V., Shushunova, N., \& Zubkova, J. Application of BIM-technologies in tasks of quality management and labour safety. In MATEC Web of Conferences (Vol. 251, p. 06004). EDP Sciences. (2018).

22. Cutini, V. Lines and squares: Towards a configurational approach to the morphology of open spaces. Proceedings of the $4^{\text {th }}$ International Space Syntax Symposium, London. (2003).

23. Varoudis, T., Psarra, S. Beyond two dimensions: Architecture through three-dimensional visibility graph analysis. The Journal of Space Syntax 5(1) pp. 90-108. (2014).

24. Kagan, P. Monitoring of the development of urban areas with the use of information technology. In MATEC Web of Conferences (Vol. 193, p. 05031). EDP Sciences. (2018).

25. Vigier, T., Siret, D., Moreau, G., \& Lescop, L. Sensitive suggestion and perception of climatic effects in virtual urban environments. In Proceedings of the ACM Symposium on Applied Perception (pp. 139-139). ACM. (2013). 
26. Kholshevnikov V.V., Samoshin D.A. Parameters of Pedestrian Flow for Modeling Purposes. In: Klingsch W., Rogsch C., Schadschneider A., Schreckenberg M. (eds) Pedestrian and Evacuation Dynamics 2008. Springer, Berlin, Heidelberg. (2010).

27. Kholshchevnikov, V. V. Experimental researches of human flow in staircases of high-rise buildings. International Journal of Applied Engineering Research, 10(21), 42549-42552. (2015).

28. Nikolic, M., Bierlaire, M. Pedestrian flow characterisation based on spatio-temporal Voronoi tessellations. $15^{\text {th }}$ Swiss Transport Research Conference STRC - Ascona 15-17April (2015).

29. Chatzikonstantinou, I. A 3-Dimensional Architectural Layout Generation Procedure for Optimization Applications: DC-RVD. Proceedings of the 32nd International Conference on Education and research in Computer Aided Architectural Design in Europe, 287-296. Vol. 1. eCAADe: Conferences 1. Newcastle upon Tyne, UK: Northumbria University, (2014).

30. Goldsmith, N. The physical modeling legacy of Frei Otto. International Journal of Space Structures, 31(1), 25-30. (2016).

31. Martinez-Gil, F., Lozano, M., Garcia-Fernandez, i., Fernandez, F. Modeling, evaluation, and scale on artificial pedestrians: A literature review. ACM Computing Surveys 50(5) article 72 (2017).

32. Leach, N. Swarm urbanism. Architectural design, 79(4), 56-63. (2009).

33. Chan. A., Lin. Y., Taipei. Ant Urbanism, Neil Leach (ed.), Digital Cities, London: Wiley. (2009)

34. Vlasov, D., \& Shirokaya, N. Development of a polyfunctional structure of transport hubs in Smart City. In IOP Conference Series: Materials Science and Engineering (Vol. 365, No. 2, p. 022022). IOP Publishing. (2018).

35. Shubenkov, M., \& Blagodeteleva, O. Dixon - Arctic outpost of Russia. Architecture and Modern Information Technologies, (Special Issue), 1-1. (2012). 